\title{
Associations between methylmercury, n-3 polyunsaturated fatty acids and antinuclear antibodies in young adults from the Seychelles Child Development Study (SCDS).
}

\author{
Emeir M. McSorley ${ }^{1}$, Maria S. Mulhern ${ }^{1}$, Alison J. Yeates ${ }^{1}$, Donald Harrington ${ }^{2}$, Edwin van \\ Wijngaarden $^{2}$, Sally W. Thurston ${ }^{2}$, Todd A. Jusko ${ }^{2}$, Toni Spence ${ }^{1}$, Philip W. Davidson ${ }^{2}$, \\ Gary J. Myres ${ }^{2}$, Gene E. Watson ${ }^{2}$, Conrad F. Shamlaye ${ }^{3}$ and J.J. Strain ${ }^{1}$ \\ ${ }^{1}$ Nutrition Innovation Centre for Food and Health (NICHE), Ulster University, Coleraine, United Kingdom, \\ ${ }^{2}$ School of Medicine and Dentistry, University of Rochester, New York, USA and \\ ${ }^{3}$ Child Development Centre, Ministry of Health, Mahé, Seychelles
}

\begin{abstract}
The association of $\mathrm{MeHg}$ exposure through fish consumption on human autoimmunity remains unclear. Fish also contain n-3 long chain polyunsaturated fatty acids (LCPUFA) that are known to regulate inflammation and mitigate autoimmune disease symptoms. We studied the association of low-level exposure to methylmercury ( $\mathrm{MeHg}$ ) through fish consumption in the SCDS. We examined this association at age 19 years in the SCDS Main Cohort $(n=497)$. We measured MeHg exposure at 3 time points [prenatal, weighted average ( 6 months to 19 years) and concurrent (19 years) and LCPUFA status and a panel of 13 autoimmune markers at age 19 years. The autoimmune markers included antinuclear antibodies (ANA), anti-dsDNA and anti-RNP, and total (non-specific) immunoglobulins (Ig) IgG, IgA, and IgM. A combined ANA variable was also calculated based on being within or above reference range for any of the ANA markers; $56 \%$ of the subjects met this criterion. Multivariable regression models adjusted for prenatal MeHg, sex and waist circumference, with and without adjustment for LCPUFA, were fit for the three $\mathrm{MeHg}$ exposure metrics and each immune marker. Mean (SD) prenatal, weighted average and concurrent $\mathrm{MeHg}$ was 6.84 (4.55), 7.46 (2.82), and 10.23 (6.02) ppm, respectively. Combined ANA was positively associated with concurrent $\mathrm{MeHg}$ following adjustment for the n6:n3 LCPUFA ratio ( $\beta=0.036,95 \%$; CI: $0.001,0.073)$. Prenatal and average $\mathrm{MeHg}$ exposures were not significantly associated with any individual ANA. IgM was negatively associated with concurrent $(\beta=-0.016,95 \% \mathrm{CI}:-0.016,-0.002)$, and average $(\beta=-0.042,95 \% \mathrm{CI}:-0.042,-0.009) \mathrm{MeHg}$ exposure in the models adjusted for n-3, n-6 LCPUFA and when separately adjusted for the n6:n3 LCPUFA ratio. Total (19-year) n-3 PUFA status was negatively associated with anti-RNP $(\beta=-20.355,95 \% \mathrm{CI}:-36.89,-4.34)$ and $\mathrm{IgG}(\beta=-1.384,95 \% \mathrm{CI}:-2.682,-0.087)$. Total n-3 LCPUFA was associated with lower markers of autoimmunity. MeHg exposure at 19 years was associated with higher ANA and lower IgM but only following adjustment for LCPUFA. The clinical significance of these findings is unclear and further research is warranted to determine if these associations precede autoimmune disease development.
\end{abstract}

\section{Conflict of Interest}

There is no conflict of interst. 